\title{
DUST AND MOLECULES IN EVOLVED STARS
}

Proceedings of an International Workshop held at UMIST, Manchester, United Kingdom, 24-27 March, 1997

\author{
Edited by \\ I. CHERCHNEFF \\ Department of Physics, UMIST, Manchester, United Kingdom \\ and \\ T. J. MILLAR \\ Department of Physics, UMIST, Manchester, United Kingdom
}

Reprinted from Astrophysics and Space Science

Volume 251, Nos. 1-2, 1997

SPRINGER-SCIENCE+BUSINESS MEDIA, B.V. 
A C.I.P. Catalogue record for this book is available from the Library of Congress.

Cover: Recurring Nova T Pyxidis observed with

Hubble Space Telescope, Wide Field Planetary Camera 2.

(PRC97-29, STSci OPO, September 18, 1997.

M. Shara, R. Williams, and R. Gilmozzi.)

Printed on acid-free paper

\begin{abstract}
All Rights Reserved
(C)1998 Springer Science+Business Media Dordrecht

Originally published by Kluwer Academic Publishers in 1998

No part of the material protected by this copyright notice may be reproduced or utilized in any form or by any means, electronic or mechanical, including photocopying, recording or by any information storage and retrieval system, without written permission from the copyright owner.
\end{abstract}




\section{TABLE OF CONTENTS}

\section{LOW-MASS STARS}

A.G.G.M. TIELENS / Circumstellar PAHS and Carbon Stardust (R)

M.J. BARLOW / ISO LWS Observations of Evolved Stars and Nebulae (R)

K. JUSTTANONT, I. YAMAMURA, T. DE JONG and L.B.F.M. WATERS / ISO-SWS Spectra of AGB Stars (C)

H. OLOFSSON / Molecules in Envelopes around AGB-Stars (R)

M.J. REID and K.M. MENTEN / Shocks in the Radio Photospheres of Long Period Variable Stars (C)

KAREN WILLACY and ISABELLE CHERCHNEFF / Silicon and Sulphur in the Inner Envelope of IRO+10216 (C)

ANITA M.S. RICHARDS, R.J. COHEN and J.A. YATES / Maser Mapping and Dust Properties of Red Supergiant Winds (C)

RAFAEL BACHILLER / Chemical Evolution from the AGB to the Planetary Nebula Phase (R)

ERIC J. BAKKER / Circumstellar Molecular Spectra Towards Evolved Stars (C)

M. JURA / Disks around Post-Main Sequence Binaries (R)

M.A.T. GROENEWEGEN, P.A. WHITELOCK, C.H. SMITH and F. KERSCHBAUM / Dust Shells around Carbon Mira Variables (C)

A. BLANCO, A. BORGHESI, E. EPIFANI, S. FONTI, V. OROFINO and F. STRAFELLA / Variability of Circumstellar Emission from Dust Envelopes around Carbon Stars (C)

ERWIN SEDLMAYR / Circumstellar Dust Condensation Scenarios (R)

A.K. SPECK and M.J. BARLOW / UIR Bands in Carbon Star Spectra (C)

N.V. VOSHCHINNIKOV and V.B. IL'IN / Non-Spherical Dust Grains in the Envelopes of Late-Type Giants (C)

M. STEFFEN and R. SZCZERBA / Modeling the Long-Term Evolution of Dusty Outflows of AGB-Stars (R)

N. MAURON / Observations of Shells Around $\mu$ CEP \& CRL2688, and about Episodic Mass Loss (C)

R. SZCZERBA and M. STEFFEN / IR-Colors for Models of Post-AGB Evolution (C)

MICHELLE J. CREECH-EAKMAN and ROBERT E. STENCEL / Variation of Silicate Dust Features with Phase (C)

TAKASHI KOZASA and HISATO SOGAWA / Formation of Dust Grains in Circumstellar Envelopes of Oxygen-Rich AGB Stars (C)

D.R. ALEXANDER, F. ALLARD, A. TAMANAI and P.H. HAUSCHILDT / Grain Formation in Atmospheres of Cool Dwarfs (P) 
S. BAGNULO and J.G. DOYLE / Observations and Modelling of Spectral Energy Distributions of Asymptotic Giant Branch Stars (P)

IRMELA BUES and TURGUT ASLAN / Backwarming Effect of Molecular Opacities on the Infrared Colours of Very Cool White Dwarfs (P)

R.D.D. COSTA, D. MÉKARNIA and J. LEFÉVRE / Modelling the Dust Envelope in Symbiotic Systems: The Case of CH Cygni (P)

MERCÈ CROSAS, KARL M. MENTEN, KEN YOUNG and THOMAS G. PHILLIPS / Radiative Transfer in a Turbulent Expanding Molecular Envelope: Application to Mira (P)

S. ETOKA and A.M. LE SQUEREN / OH Maser Lines Variability of Miras (P)

P.D. GENSHEIMER / Observations of HCCNC and HNCCC in IRC+10216 (P)

P. HALL / Modelling Software for Dust Processes (P)

O. HASHIMOTO and H. IZUMIURA / A Compact Circumstellar Dust Envelope of an Evolved AGB Star IRC-10529 (P)

JOSEF HRON, RITA LOIDL and FRANZ KERSCHBAUM / Dust Features of Visually Bright AGB Variables As Seen By ISO and IRAS (P)

E.M.L. HUMPHREYS, M.D. GRAY, D. FIELD, J.A. YATES and G. BOWEN / A Model for the Variability of SIO Masers in Mira Variables (P)

M. JOHN and E. SEDLMAYR / Formation of Small Iron Clusters in Circumstellar Envelopes (P)

C. KAHANE, E. VIARD, F. MENARD, P. BASTIEN and N. MANSET / Polarization of Evolved Stars Envelopes (P)

GULCIN KANDEMIR and SENEL BOYDAG / A Simulation for a Planetary Nebular Plasma (P)

A. KUČINSKAS / Terminal Outflow Velocities from the Mass-Losing AGB Stars (P)

F. LARSEN, H. OLOFSSON, K. ERIKSSON and B. GUSTAFSSON / Molecules in Circumstellar Envelopes of Carbon Stars (P)

T. LLOYD EVANS / Stars With Cool Dusty Disks (P)

RITA LOIDL, JOSEF HRON, SUSANNE HÖFNER, UFFE G. JØRGENSEN, BERNHARD ARINGER and FRANZ KERSCHBAUM / Synthetic Spectra of Long-Period Variables: A First Comparison With ISO Observations (P)

R. LUCAS / 1.3 MM Plateau De Bure Observations of IRC+10216 (P)

M. MARENGO, M. BUSSO, P. PERSI, P.O. LAGAGE and G. SILVESTRO / MID-IR Imaging and Modelling of AGB Circumstellar Envelopes (P)

A.J. MARKWICK and T.J. MILLAR / Carbon-Bearing Molecules in OxygenRich Circumstellar Envelopes (P)

G.M. RUDNITSKIJ / Effects of Dust on the Propagation of Shock Waves in Circumstellar Envelopes of Late-Type Variable Stars (P1) 
G.M. RUDNITSKIJ / Models of Circumstellar Maser in Late-Type Variable Stars (P2)

A.V. SHAVRINA and L.A. YAKOVINA / On the Molecular Line Absorption in the LI I Doublet $6708 \AA$ Region for Evolved Star Atmospheres (P)

ROGER J. SYLVESTER / Detection of the Far-IR Pumping Lines for OH Masers (P)

S.D. TAYLOR and D.A. WILLIAMS / Molecules in Boundary Layers in Planetary Nebulae (P)

KAREN WILLACY and T.J. MILLAR / Carbon Chemistry in Oxygen-Rich Circumstellar Envelopes (P)

J.A. YATES, E.M.L. HUMPHREYS and A.M.S. RICHARDS / Simulating $\mathrm{H}_{2} \mathrm{O}$ Maser Emission in the Mass Outflows from Evolved Stars (P)

LAIMONS ZAČS / Detection of $\mathrm{C}_{2}$ and $\mathrm{C}_{3}$ Absorption in Two Post-AGB Objects IRAS 22272+5435 and CIT 6 (P)

\section{NOVAE, HIGH MASS STARS}

A. EVANS / Dust and Molecules in Nova Environments (R)

S.P.S. EYRES, A. EVANS, T.R. GEBALLE, J.K. DAVIES and J.M.C. RAWLINGS / Dust Evolution in Nova Cassiopeia 1993 (C)

KAREL A. VAN DER HUCHT / Physical Properties of Wolf-Rayet Winds (R)

P.M. WILLIAMS / Formation of Dust in Hostile Environments - What We Learn From Observing Wolf-Rayet Stars (R)

ISABELLE CHERCHNEFF / Dust in Hydrogen-Poor Stellar Winds, From Theory to Observations (R)

V.F. POLCARO and L. NORCI / V 439 CYG, The Red Star That Became Blue (C)

LINDA J. SMITH / The Origin of the Nebulae Surrounding Luminous Blue Variable Stars $(C)$

DONALD D. CLAYTON, SACHIKO AMARI and ERNST ZINNER / Dust from Supernovae (R)

A. DALGARNO, P.C. STANCIL and S. LEPP / Molecules and Dust in Supernovae (R)

M.E.L. HOPWOOD, A. EVANS, S.P.S. EYRES and A. PENNY / A Search For CO $J=2 \rightarrow 1$ Emission From the Metal Rich Globular Cluster NGC $6356(\mathrm{P})$

YOANN H. LE TUEFF and ISABELLE CHERCHNEFF / Dust Formation Chemistry in Carbon-Rich Wolf-Rayet Winds (P)

Index of Objects

Index of Topics 


\section{PREFACE}

The Workshop recorded in this volume was held at UMIST in March 1997 and was entitled 'Dust and Molecules in Evolved Stars'. Following the tradition of the UMIST group to organize regular conference series on topics related to the expertise of the group, namely Astrochemistry, the Workshop focused principally on observational and theoretical aspects of the study of chemical species, from molecules to dust grains, in the circumstellar environments of evolved objects. Our aim was to gather together various scientific communities, generally unknown to each other, and working on different types of evolved stars, to address fundamental questions on molecule and dust formation and survival, observational diagnostic tools common to evolved astrophysical environments, and so on. The astrophysical scenarios included late-type stars, novae, WolfRayet stars, LBVs and supernovae, and it was often highlighted that similar chemical entities, dust or molecules, were found in environments characterized by very different physical and chemical parameters. Therefore, there was a real need to bridge the gap between the various communities in order to shed light on these mysteries. At the time of the Workshop new results from the ISO satellite had arrived and the participants were able to benefit from these new data in the context of the conference.

The Workshop was attended by more than 80 scientists coming from 17 different countries, reflecting the fact that the study of evolved stars, both from observation and theory, represents a very active field of Astrophysics and Astrochemistry.

This volume contains all the review papers (denoted $\mathrm{R}$ ) but one. Unfortunately, Peter Sarre was not able to provide a manuscript. As editors, we would like to express our gratitude to the reviewers who strove hard to restrict themselves to the allocated length for, their manuscripts. We have also included the contributed papers (denoted C) and the poster papers (denoted P). Discussions have been inserted at the end of each $R$ and $C$ paper. Unfortunately, the vivid discussions accompanying the poster sessions could not be recorded.

The Workshop was supported financially by various sponsors. We are very grateful to the Collaborative Computational Project No. 7 on 'The Analysis of Astronomical Spectra', the Royal Astronomical Society, the Astrophysics Group in the Department of Physics at UMIST, our publisher, Kluwer, and the European Union. The latter sponsored the attendance of several of our colleagues from Eastern Europe and the new Independent States of the Soviet Union.

I. Cherchneff, T. J. Millar UMIST

November 1997 
ALEXANDER David R.

\section{BACHILLER Rafael}

BAGNULO Stefano

BAKKER Eric J.

BARLOW Mike J.

BUES Irmela

CHERCHNEFF Isabelle

CLAYTON Donald D.

COHEN Martin

COSTA Roberto D.D.

CREECH-EAKMAN Michelle J.

CROSAS Merce

DALGARNO Alexander

ETOKA Sandra

EVANS Nye

EYRES Stewart P.

FLOWER David

FONTI, Sergio

GENSHEIMER Paul

GROENEWEGEN Martin

HALL Peter F.

HASHIMOTO Osamu

HATCHELL Jennifer J.

HOPWOOD Madelaine E.

HOWE David A.

HUMPHREYS Elizabeth

\section{LIST OF PARTICIPANTS}

Dept. of Physics, Wichita State University, Wichita, USA

dra@twsuvm.uc.twsu.edu

Observatorio Astronomico Nacional, Alcala de Henares, Spain

bachiller@conga.oan.es

Armagh Observatory, Armagh, Northern Ireland, UK

sba@star.arm.ac.uk

Dept. of Astronomy, University of Texas, Austin, USA

ebakker@viking.as.utexas.edu

Dept. of Physics \& Astronomy, University College London, London, UK mjb@star.ucl.ac.uk

Astron. Institut, Universitaet Erlangen-Nuernberg, Bamberg, Germany bues@sternwarte.uni-erlangen.de

Dept. of Physics, UMIST, Manchester, UK

imc@europa.phy.umist.ac.uk

Dept. of Physics and Astronomy, Clemson University, Clemson, USA

clayton@gamma.phys.clemson.edu

Radio Astronomy Laboratory, University of California, Berkeley, USA

mcohen@astro.berkeley.edu

Observatoire de la Cote d'Azur, Nice, France

costa@obs-nice.fr

Dept. of Physics \& Astronomy, University of Denver, Denver, USA

mce@phoenix.phys.du.edu

Harvard-Smithsonian Center for Astrophysics, Cambridge, USA

mcrosas@cfa.harvard.edu

Harvard-Smithsonian Center for Astrophysics, Cambridge, USA

adalgarno@cfa.harvard.edu

Torun Radio Astronomy Obs., N. Copernicus University, Torun, Poland sandra@newton.astro.uni.torun.pl

Dept. of Physics, University of Keele, Keele, UK

ae@astro.keele.ac.uk

Dept. of Physics, University of Keele, Keele, UK

spe@astro.keele.ac.uk

Dept. of Physics, University of Durham, Durham, UK

david.flower@dur.ac.uk

Dept. of Physics, University of Lecce, Lecce, Italy

fonti@le.infn.it

Max-Planck-Institut fuer Radioastronomie, Bonn, Germany paulg@mpifr-bonn.mpg.de

Max-Planck-Institut fuer Astrophysik, Garching, Germany

groen@mpa-garching.mpg.de

Dept. of Physics, UMIST, Manchester, UK

pfh@saturn.umist.ac.uk

Dept. of Applied Physics, University of Seikei, Tokyo, Japan

hasimoto@aps.seikei.ac.jp

Dept. of Physics, UMIST, Manchester, UK

jjh@saturn.phy.umist.ac.uk

Dept. of Physics, University of Keele, Keele, UK

meh@astro.keele.ac.uk

Dept. of Physics, UMIST, Manchester, UK

dah@saturn.phy.umist.ac.uk

School of Chemistry, University of Bristol, Bristol, UK

liz.humphreys@bristol.ac.uk 
IDIART Thais E.P.

JEFFERY C. Simon

JOHN Marcus

JURA Michael

JUSTTANONT Kay

KAHANE Claudine

KANDEMIR Gulcin

KOZASA Takashi

KUCINSKAS Arunas

LARSEN Fredrik

LE TEUFF Yoann H.

LIBERATORE Stephane

LIU X.-W.

LLOYD EVANS Tom

LOIDL Rita

LUCAS Robert

MARENGO Massimo

MARKWICK Andrew J.

MAURON Nicolas

MILLAR Tom J.

OLOFSSON Hans

POLCARO Vita F.

PONTEFRACT Matthew

REID Mark J.

RICHARDS Anita M.S.

RODGERS Steven D.
Observatoire de la Cote d'Azur, Nice, France

thais@obs-nice.fr

Armagh Observatory, Armagh, Northern Ireland, UK

csj@star.arm.ac.uk

Inst. fuer Astronomie und Astrophysik, Tech. Universitaet Berlin, Germany

john@pils.physik.TU-Berlin.de

Dept. of Astronomy, UCLA, Los Angeles, USA

jura@clotho.astro.ucla.edu

SRON, Groningen, The Netherlands

kay@sron.rug.nl

Laboratoire d'Astrophysique Observatoire de Grenoble, Grenoble, France kahane@gag.observ-gr.fr

Dept. of Physics, Istanbul technical University, Istanbul, Turkey

kandemir@sariyer.cc.itu.edu.tr

Dept. of Earth \& Planetary Sciences, Kobe University, Kobe, Japan

kozasa@kobe-u.ac.jp

Institute of Theoretical Physics \& Astronomy, Vilnius, Lithuania

karunas@pub.osf.lt

Stockholm Observatory, Saltsjobaden, Sweden

fredrik@astro.su.se

Dept. of Physics, UMIST, Manchester, UK

ylt@saturn.phy.umist.ac.uk

Observatoire de Paris-Meudon, DASGAL, Meudon, France

liberatore@obspm.fr

Dept. of Physics \& Astronomy, University College London, London, UK xwl@star.ucl.ac.uk

South African Astronomical Observatory, Cape, South Africa

tle@saao.ac.za

Turkenschanzstrasse 17, Wien, Austria

loidl@astro.ast.univie.ac.at

Institut de Radioastronomie Millimetrique, St. Martin d'Heres, France

lucas@iram.fr

SISSA/ISAS International School for Advanced Studies, Trieste, Italy

marengo@sissa.it

Dept. of Physics, UMIST, Manchester, UK

ajm@saturn.phy.umist.ac.uk

GRAAL, University de Montpellier II, Montpellier, France

mauron@graal.univ-montp2.fr

Dept. of Physics, UMIST, Manchester, UK

tjm@saturn.phy.umist.ac.uk

Stockholm Observatory, Saltsjobaden, Sweden

hans@astro.su.se

Instituto di Astrofisica Spaziale, V. Enrico Fermi, Italy

polcaro@saturn.ias.fra.cnr.it

Dept. of Physics \& Astronomy, University College London, London, UK mp@star.ucl.ac.uk

Harvard-Smithsonian Center for Astrophysics, Cambridge, USA

mreid@cfa.harvard.edu

NRAL, Jodrell Bank, Macclesfield, UK

amsr@jb.man.ac.uk

Dept. of Physics, UMIST, Manchester, UK

sdr@saturn.phy.umist.ac.uk 
RUDNITSKIJ Georgij

SARRE Peter

SCHROEDER Klaus-Peter

SEDLMAYR Erwin

SHAVRINA Angelina V.

SMITH Linda J.

SPECK Angela

STEFFEN Matthias

SYLVESTER Roger

SZCZERBA Ryszard

TAMANAI Akemi

TAYLOR Stephen D.

TIELENS Alexander G.G.M.

TSIOPA Olga

van der HUCHT Karel A.

VOSHCHINNIKOV Nikolai

WAGENBLAST Ralf $M$.

WALKER Helen J.

WATSON Sarah K.

WILLACY Karen A.

WILLIAMS David A.

WILLIAMS Peredur M.

YATES Jeremy

ZACS Laimons
Sternberg Astronomical Institute, Moscow, Russia

gmr@sai.msu.su

Chemistry Department, University of Nottingham, Nottingham, UK psa@star.le.ac.uk

Inst. fuer Astronomie und Astrophysik, Tech. Universitaet Berlin, Germany schroder@physik.tu-berlin.de

Inst. fuer Astronomie und Astrophysik, Tech. Universitaet Berlin, Germany sedlmayr@physik.tu-berlin.de

Main Astron. Obs. of Acad. of Science of Ukraine, Golosiev Kiev, Ukraine shavrina@mao.kiev.ua

Dept. of Physics \& Astronomy, University College London, London, UK ljs@star.ucl.ac.uk

Dept. of Physics \& Astronomy, University College London, London, UK aks@star.ucl.ac.uk

Astrophysikalisches Institut Potsdam, Potsdam, Germany

msteffen@aip.de

Dept. of Physics \& Astronomy, University College London, London, UK rjs@star.ucl.ac.uk

N. Copernicus Astronomical Center, Torun, Poland

szczerba@ncac.torun.pl

Department of Physics, Wichita State University, Wichita, USA

tamanai@twsu.campus.mci.net

Dept. of Physics \& Astronomy, University College London, London, UK sdt@star.ucl.ac.uk

NASA/Ames Research Center, Moffett Field, USA

tielens@dusty.arc.nasa.gov

Central Astron. Obs. of the Russian Acad. of Sci., St. Petersburg, Russia tsiopa@pulkovo.spb.su

Space Research Organization Netherlands, Utrecht, The Netherlands

k.vanderhucht@sron.ruu.nl

Astronomical Institute, St. Petersburg, Russia

nvv@aispbu.spb.su

Dept. of Physics, UMIST, Manchester, UK

r.wagenblast@umist.ac.uk

Rutherford Appleton Laboratory, Didcot, UK

h.walker@rl.ac.uk

NRAL, Jodrell Bank, Macclesfield, UK

skw@jb.man.ac.uk

Dept. of Physics, UMIST, Manchester, UK

kaw@saturn.phy.umist.ac.uk

Dept. of Physics \& Astronomy, University College London, London, UK daw@star.ucl.ac.uk

Royal Observatory, Edinburgh, UK

pmw@roe.ac.uk

Dept. of Physics, University of Hertfordshire, Hatfield, UK

jyates@star. herts.ac.uk

Radioastrophysical Observatory, Baldone, Latvia

zacs@acad.latnet.lv 